\title{
EXTENSION OF HOLOMORPHIC FUNCTIONS AND COHOMOLOGY CLASSES FROM NON REDUCED ANALYTIC SUBVARIETIES
}

\author{
JEAN-PIERRE DEMAILLY* \\ in honor of Professor Kang-Tae Kim on the occasion of his sixtieth birthday
}

\begin{abstract}
The goal of this survey is to describe some recent results concerning the $L^{2}$ extension of holomorphic sections or cohomology classes with values in vector bundles satisfying weak semi-positivity properties. The results presented here are generalized versions of the OhsawaTakegoshi extension theorem, and borrow many techniques from the long series of papers by $\mathrm{T}$. Ohsawa. The recent achievement that we want to point out is that the surjectivity property holds true for restriction morphisms to non necessarily reduced subvarieties, provided these are defined as zero varieties of multiplier ideal sheaves. The new idea involved to approach the existence problem is to make use of $L^{2}$ approximation in the Bochner-Kodaira technique. The extension results hold under curvature conditions that look pretty optimal. However, a major unsolved problem is to obtain natural (and hopefully best possible) $L^{2}$ estimates for the extension in the case of non reduced subvarieties - the case when $Y$ has singularities or several irreducible components is also a substantial issue.
\end{abstract}

\section{INTRODUCTION AND MAIN RESULTS}

The problem considered in these notes is whether a holomorphic object $f$ defined on a subvariety $Y$ of a complex manifold $X$ can be extended as a holomorphic object $F$ of the same nature on the whole of $X$. Here, $Y$ is a subvariety defined as the the zero zet of a non necessarily reduced ideal $\mathcal{I}$ of $\mathcal{O}_{X}$, the object to extend can be either a section $f \in H^{0}\left(Y, E_{\mid Y}\right)$ or a cohomology class $f \in H^{q}\left(Y, E_{\| Y}\right)$, and we look for an extension $F \in H^{q}(X, E)$, assuming suitable convexity properties of $X$ and $Y$, suitable $L^{2}$ conditions for $f$ on $Y$, and appropriate curvature positivity hypotheses for the bundle $E$. When $Y$ is not connected, this can be also seen as an interpolation problem - the situation where $Y$ is a discrete set is already very interesting.

The prototype of such results is the celebrated $L^{2}$ extension theorem of Ohsawa-Takegoshi OT87, which deals with the important case when $X=\Omega \subset \mathbb{C}^{n}$ is a pseudoconvex open set, and $Y=\Omega \cap L$ is the intersection of $\Omega$ with a complex affine linear subspace $L \subset \mathbb{C}^{n}$. The accompanying $L^{2}$ estimates play a very important role in applications, possibly even more than the qualitative extension theorems by themselves (cf. section 4 below). The related techniques have then been the subject of many works since 1987, proposing either greater generality ( Ohs88, Ohs94, Ohs95, Ohs01, Ohs03, Ohs05], [Man93], [Dem97], Pop05]), alternative proofs ([Ber96], Che11]), improved estimates ([MV07], [Var10]) or optimal ones ([Blo13], GZ15a], [BL16]).

In this survey, we mostly follow the lines of our previous papers [Dem15b] and [CDM17, whose goal is to pick the weakest possible curvature and convexity hypotheses, while allowing the subvariety $Y$ to be non reduced. The ambient complex manifold $X$ is assumed to be a Kähler and holomorphically convex (and thus not necessarily compact); by the Remmert reduction theorem, the holomorphic convexity is equivalent to the existence of a proper holomorphic map

Date: May 29, 2022.

2010 Mathematics Subject Classification. Primary 32L10; Secondary 32E05.

Key words and phrases. Compact Kähler manifold, singular hermitian metric, coherent sheaf cohomology, Dolbeault cohomology, plurisubharmonic function, $L^{2}$ estimates, Ohsawa-Takegoshi extension theorem, multiplier ideal sheaf.

Supported by the European Research Council project "Algebraic and Kähler Geometry" (ERC-ALKAGE, grant No. 670846 from September 2015). 
$\pi: X \rightarrow S$ onto a Stein complex space $S$, hence arbitrary relative situations over Stein bases are allowed. We consider a holomorphic line bundle $E \rightarrow X$ equipped with a singular hermitian metric $h$, namely a metric which can be expressed locally as $h=e^{-\varphi}$ where $\varphi$ is a quasi-psh function, i.e. a function that is locally the $\operatorname{sum} \varphi=\varphi_{0}+u$ of a plurisubharmonic function $\varphi_{0}$ and of a smooth function $u$. Such a bundle admits a curvature current

$$
\Theta_{E, h}:=i \partial \bar{\partial} \varphi=i \partial \bar{\partial} \varphi_{0}+i \partial \bar{\partial} u
$$

which is locally the sum of a positive $(1,1)$-current $i \partial \bar{\partial} \varphi_{0}$ and a smooth $(1,1)$-form $i \partial \bar{\partial} u$. Our goal is to extend sections that are defined on a non necessarily reduced complex subspace $Y \subset X$, when the structure sheaf $\mathcal{O}_{Y}:=\mathcal{O}_{X} / \mathcal{I}\left(e^{-\psi}\right)$ is given by the multiplier ideal sheaf of a quasi-psh function $\psi$ with neat analytic singularities, i.e. locally on a neighborhood $V$ of an arbitrary point $x_{0} \in X$ we have

$$
\psi(z)=c \log \sum\left|g_{j}(z)\right|^{2}+v(z), \quad g_{j} \in \mathcal{O}_{X}(V), \quad v \in C^{\infty}(V) .
$$

Let us recall that the multiplier ideal sheaf $\mathcal{I}\left(e^{-\varphi}\right)$ of a quasi-psh function $\varphi$ is defined by

$$
\mathcal{I}\left(e^{-\varphi}\right)_{x_{0}}=\left\{f \in \mathcal{O}_{X, x_{0}} ; \exists U \ni x_{0}, \int_{U}|f|^{2} e^{-\varphi} d \lambda<+\infty\right\}
$$

with respect to the Lebesgue measure $\lambda$ in some local coordinates near $x_{0}$. As is well known, $\mathcal{I}\left(e^{-\varphi}\right) \subset \mathcal{O}_{X}$ is a coherent ideal sheaf (see e.g. Dem-book]). We also denote by $K_{X}=\Lambda^{n} T_{X}^{*}$ the canonical bundle of an $n$-dimensional complex manifold $X$; in the case of (semi)positive curvature, the Bochner-Kodaira identity yields positive curvature terms only for $(n, q)$-forms, so the best way to state results is to consider the adjoint bundle $K_{X} \otimes E$ rather than the bundle $E$ itself. The main qualitative statement is given by the following result of [CDM17].

Theorem 1.1. Let $E$ be a holomorphic line bundle over a holomorphically convex Kähler manifold $X$. Let $h$ be a possibly singular hermitian metric on $E, \psi$ a quasi-psh function with neat analytic singularities on $X$. Assume that there exists a positive continuous function $\delta>0$ on $X$ such that

$$
\Theta_{E, h}+(1+\alpha \delta) i \partial \bar{\partial} \psi \geq 0 \quad \text { in the sense of currents, for } \alpha=0,1 \text {. }
$$

Then the morphism induced by the natural inclusion $\mathcal{I}\left(h e^{-\psi}\right) \rightarrow \mathcal{I}(h)$

$$
H^{q}\left(X, K_{X} \otimes E \otimes \mathcal{I}\left(h e^{-\psi}\right)\right) \rightarrow H^{q}\left(X, K_{X} \otimes E \otimes \mathcal{I}(h)\right)
$$

is injective for every $q \geq 0$. In other words, the morphism induced by the natural sheaf surjection $\mathcal{I}(h) \rightarrow \mathcal{I}(h) / \mathcal{I}\left(h e^{-\psi}\right)$

$$
H^{q}\left(X, K_{X} \otimes E \otimes \mathcal{I}(h)\right) \rightarrow H^{q}\left(X, K_{X} \otimes E \otimes \mathcal{I}(h) / \mathcal{I}\left(h e^{-\psi}\right)\right)
$$

is surjective for every $q \geq 0$.

Remark 1.2. (A) When $h$ is smooth, we have $\mathcal{I}(h)=\mathcal{O}_{X}$ and

$$
\mathcal{I}(h) / \mathcal{I}\left(h e^{-\psi}\right)=\mathcal{O}_{X} / \mathcal{I}\left(e^{-\psi}\right):=\mathcal{O}_{Y}
$$

where $Y$ is the zero subvariety of the ideal sheaf $\mathcal{I}\left(e^{-\psi}\right)$. Hence, the surjectivity statement can be interpreted an extension theorem with respect to the restriction morphism

$$
H^{q}\left(X, K_{X} \otimes E\right) \rightarrow H^{q}\left(Y,\left(K_{X} \otimes E\right)_{\mid Y}\right) .
$$

In general, the quotient sheaf $\mathcal{I}(h) / \mathcal{I}\left(h e^{-\psi}\right)$ is supported in an analytic subvariety $Y \subset X$, which is the zero set of the conductor ideal

$$
\mathcal{J}_{Y}:=\mathcal{I}\left(h e^{-\psi}\right): \mathcal{I}(h)=\left\{f \in \mathcal{O}_{X} ; f \cdot \mathcal{I}(h) \subset \mathcal{I}\left(h e^{-\psi}\right)\right\},
$$

and (1.6) can thus also be considered as a restriction morphism.

(B) A surjectivity statement similar to (1.7) holds true when $(E, h)$ is a holomorphic vector bundle equipped with a smooth hermitian metric $h$. In that case, the required curvature condition (1.4) is a semipositivity assumption

$$
\Theta_{E, h}+(1+\alpha \delta) i \partial \bar{\partial} \psi \otimes \operatorname{Id}_{E} \geq 0 \quad \text { in the sense of Nakano, for } \alpha=0,1
$$


(This means that the corresponding hermitian form on $T_{X} \otimes E$ takes nonnegative values on all tensors of $T_{X} \otimes E$, even those that are non decomposable.)

(C) The strength of our statements lies in the fact that no strict positivity assumption is made. This is a typical situation in algebraic geometry, e.g. in the study of the minimal model program (MMP) for varieties which are not of general type. Our joint work [DHP13] contains some algebraic applications which we intend to reinvestigate in future work, by means of the present stronger qualitative statements.

(D) Notice that if one replaces (1.4) by a strict positivity hypothesis

$$
\Theta_{E, h}+i \partial \bar{\partial} \psi \geq \varepsilon \omega \quad \text { in the sense of currents, for some } \varepsilon>0,
$$

then Nadel's vanishing theorem implies $H^{q}\left(X, \mathcal{O}_{X}\left(K_{X} \otimes E\right) \otimes \mathcal{I}\left(h e^{-\psi}\right)\right)=0$ for $q \geq 1$, and the injectivity and surjectivity statements are just trivial consequences.

(E) By applying convex combinations, one sees that condition (1.4) takes an equivalent form if we assume the inequality to hold for $\alpha$ varying in the whole interval $[0,1]$.

We now turn ourselves to the problem of establishing $L^{2}$ estimates for the extension problem, along the lines of [OT87. The reader will find all details in Dem15b.

Definition 1.3. If $\psi$ is a quasi-psh function on a complex manifold $X$, we say that the singularities of $\psi$ are $\log$ canonical along the zero variety $Y=V\left(\mathcal{I}\left(e^{-\psi}\right)\right)$ if $\mathcal{I}\left(e^{-(1-\varepsilon) \psi}\right)_{\mid Y}=\mathcal{O}_{X\lceil Y}$ for every $\varepsilon>0$.

In case $\psi$ has $\log$ canonical singularities, it is easy to see by the Hölder inequality and the result of Guan-Zhou GZ15b] on the "strong openness conjecture" that $\mathcal{I}(\psi)$ is a reduced ideal, i.e. that $Y=V(\mathcal{I}(\psi))$ is a reduced analytic subvariety of $X$. If $\omega$ is a Kähler metric on $X$, we let $d V_{X, \omega}=\frac{1}{n !} \omega^{n}$ be the corresponding Kähler volume element, $n=\operatorname{dim} X$. In case $\psi$ has $\log$ canonical singularities along $Y=V(\mathcal{I}(\psi))$, one can also associate in a natural way a measure $d V_{Y^{\circ}, \omega}[\psi]$ on the set $Y^{\circ}=Y_{\text {reg }}$ of regular points of $Y$ as follows. If $g \in \mathcal{C}_{c}\left(Y^{\circ}\right)$ is a compactly supported continuous function on $Y^{\circ}$ and $\widetilde{g}$ a compactly supported extension of $g$ to $X$, we set

$$
\int_{Y^{\circ}} g d V_{Y^{\circ}, \omega}[\psi]=\limsup _{t \rightarrow-\infty} \int_{\{x \in X, t<\psi(x)<t+1\}} \widetilde{g} e^{-\psi} d V_{X, \omega} .
$$

By the Hironaka desingularization theorem, one can show that the limit does not depend on the continuous extension $\widetilde{g}$, and that one gets in this way a measure with smooth positive density with respect to the Lebesgue measure, at least on an (analytic) Zariski open set in $Y^{\circ}$. In case $Y$ is a codimension $r$ subvariety of $X$ defined by an equation $\sigma(x)=0$ associated with a section $\sigma \in H^{0}(X, S)$ of some hermitian vector bundle $\left(S, h_{S}\right)$ on $X$, and assuming that $\sigma$ is generically transverse to zero along $Y$, it is natural to take

$$
\psi(z)=r \log |\sigma(z)|_{h_{S}}^{2} .
$$

One can then easily check that $d V_{Y^{\circ}, \omega}[\psi]$ is the measure supported on $Y^{\circ}=Y_{\text {reg }}$ such that

$$
d V_{Y^{\circ}, \omega}[\psi]=\frac{2^{r+1} \pi^{r}}{(r-1) !} \frac{1}{\left|\Lambda^{r}(d \sigma)\right|_{\omega, h_{S}}^{2}} d V_{Y, \omega} \quad \text { where } \quad d V_{Y, \omega}=\frac{1}{(n-r) !} \omega_{\mid Y^{\circ}}^{n-r} .
$$

For a quasi-psh function with log canonical singularities, $d V_{Y^{\circ}, \omega}[\psi]$ should thus be seen as some sort of (inverse of) Jacobian determinant associated with the logarithmic singularities of $\psi$. In general, the measure $d V_{Y^{\circ}, \omega}[\psi]$ blows up (i.e. has infinite volume) in a neighborhood of singular points of $Y$. Finally, the following positive real function will make an appearance in several of our estimates :

$$
\gamma(x)=\exp (-x / 2) \text { if } x \geq 0, \quad \gamma(x)=\frac{1}{1+x^{2}} \text { if } x \leq 0 .
$$

The first generalized $L^{2}$ estimate we are interested in is a variation of Theorem 4 in [Ohs01]. One difference is that we do not require any specific behavior of the quasi-psh function $\psi$ defining the subvariety: any quasi-psh function with log canonical singularities will do; secondly, we do not want to make any assumption that there exist negligible sets in the ambient manifold 
whose complements are Stein, because such an hypothesis need not be true on a general compact Kähler manifold - one of the targets of our study.

Theorem 1.4 ( $L^{2}$ estimate for the extension from reduced subvarieties). Let $X$ be a holomorphically convex Kähler manifold, and $\omega$ a Kähler metric on $X$. Let $(E, h)$ be a holomorphic vector bundle equipped with a smooth hermitian metric $h$ on $X$, and let $\psi: X \rightarrow[-\infty,+\infty[$ be a quasi-psh function on $X$ with neat analytic singularities. Let $Y$ be the analytic subvariety of $X$ defined by $Y=V\left(\mathcal{I}\left(e^{-\psi}\right)\right)$ and assume that $\psi$ has $\log$ canonical singularities along $Y$, so that $Y$ is reduced. Finally, assume that the Chern curvature tensor $\Theta_{E, h}$ is such that the sum

$$
\Theta_{E, h}+(1+\alpha \delta) i \partial \bar{\partial} \psi \otimes \operatorname{Id}_{E}
$$

is Nakano semipositive for some $\delta>0$ and $\alpha=0,1$. Then for every holomorphic section $f \in H^{0}\left(Y^{\circ},\left(K_{X} \otimes E\right)_{\mid Y^{\circ}}\right)$ on $Y^{\circ}=Y_{\text {reg }}$ such that

$$
\int_{Y^{\circ}}|f|_{\omega, h}^{2} d V_{Y^{\circ}, \omega}[\psi]<+\infty
$$

there exists an extension $F \in H^{0}\left(X, K_{X} \otimes E\right)$ whose restriction to $Y^{\circ}$ is equal to $f$, such that

$$
\int_{X} \gamma(\delta \psi)|F|_{\omega, h}^{2} e^{-\psi} d V_{X, \omega} \leq \frac{34}{\delta} \int_{Y^{\circ}}|f|_{\omega, h}^{2} d V_{Y^{\circ}, \omega}[\psi] .
$$

Remark 1.5. Although $|F|_{\omega, h}^{2}$ and $d V_{X, \omega}$ both depend on $\omega$, it is easy to see that the product $|F|_{\omega, h}^{2} d V_{X, \omega}$ actually does not depend on $\omega$ when $F$ is a $(n, 0)$-form. The same observation applies to the product $|f|_{\omega, h}^{2} d V_{Y^{\circ}, \omega}[\psi]$, hence the final $L^{2}$ estimate is in fact independent of $\omega$. Nevertheless, the existence of a Kähler metric (and even of a complete Kähler metric) is crucial in the proof, thanks to the techniques developped in [AV65] and [Dem82]. The constant 34 is of course non optimal; the technique developed in GZ15a provides optimal choices of the function $\gamma$ and of the constant in the right hand side.

We now turn ourselves to the case where non reduced multiplier ideal sheaves and non reduced subvarieties are considered. This situation has already been considered by D. Popovici [Pop05] in the case of powers of a reduced ideal, but we aim here at a much wider generality, which also yields more natural assumptions. For $m \in \mathbb{R}_{+}$, we consider the multiplier ideal sheaf $\mathcal{I}\left(e^{-m \psi}\right)$ and the associated non necessarily reduced subvariety $Y^{(m)}=V\left(\mathcal{I}\left(e^{-m \psi}\right)\right)$, together with the structure sheaf $\mathcal{O}_{Y^{(m)}}=\mathcal{O}_{X} / \mathcal{I}\left(e^{-m \psi}\right)$, the real number $m$ being viewed as some sort of multiplicity - the support $\left|Y^{(m)}\right|$ may increase with $m$, but certainly stabilizes to the set of poles $P=\psi^{-1}(-\infty)$ for $m$ large enough. We assume the existence of a discrete sequence of positive numbers

$$
0=m_{0}<m_{1}<m_{2}<\ldots<m_{p}<\ldots
$$

such that $\mathcal{I}\left(e^{-m \psi}\right)=\mathcal{I}\left(e^{-m_{p} \psi}\right)$ for $m \in\left[m_{p}, m_{p+1}\left[\right.\right.$ ( with of course $\left.\mathcal{I}\left(e^{-m_{0} \psi}\right)=\mathcal{O}_{X}\right)$; they are called the jumping numbers of $\psi$. The existence of a discrete sequence of jumping numbers is automatic if $X$ is compact. In general, this still holds on every relatively compact open subset

$$
X_{c}:=\{x \in X, \rho(x)<c\} \Subset X,
$$

but requires some some of uniform behaviour of singularities at infinity in the non compact case. We are interested in extending a holomorphic section

$$
f \in H^{0}\left(Y^{\left(m_{p}\right)}, \mathcal{O}_{Y^{\left(m_{p}\right)}}\left(K_{X} \otimes E_{\mid Y^{\left(m_{p}\right)}}\right):=H^{0}\left(Y^{\left(m_{p}\right)}, \mathcal{O}_{X}\left(K_{X} \otimes_{\mathbb{C}} E\right) \otimes_{\mathcal{O}_{X}} \mathcal{O}_{X} / \mathcal{I}\left(e^{-m_{p} \psi}\right)\right) .\right.
$$

[Later on, we usually omit to specify the rings over which tensor products are taken, as they are implicit from the nature of objects under consideration]. The results are easier to state in case one takes a nilpotent section of the form

$$
f \in H^{0}\left(Y^{\left(m_{p}\right)}, \mathcal{O}_{X}\left(K_{X} \otimes E\right) \otimes \mathcal{I}\left(e^{-m_{p-1} \psi}\right) / \mathcal{I}\left(e^{-m_{p} \psi}\right)\right) .
$$

Then $\left.\mathcal{I}\left(e^{-m_{p-1} \psi}\right) / \mathcal{I}\left(e^{-m_{p} \psi}\right)\right)$ is actually a coherent sheaf, and one can see that its support is a reduced subvariety $Z_{p}$ of $Y^{\left(m_{p}\right)}$. Therefore $\left.\mathcal{I}\left(e^{-m_{p-1} \psi}\right) / \mathcal{I}\left(e^{-m_{p} \psi}\right)\right)$ can be seen as a vector bundle over a Zariski open set $Z_{p}^{\circ} \subset Z_{p}$. We can mimic formula (1.11) and define some sort of 
infinitesimal " $m_{p}$-jet" $L^{2}$ norm $\left|J^{m_{p}} f\right|_{\omega, h}^{2} d V_{Z_{p}^{\circ}, \omega}[\psi]$ (a purely formal notation), as the measure on $Z_{p}^{\circ}$ defined by

$$
\int_{Z_{p}^{\circ}} g\left|J^{m_{p}} f\right|_{\omega, h}^{2} d V_{Z_{p}^{\circ}, \omega}[\psi]=\limsup _{t \rightarrow-\infty} \int_{\{x \in X, t<\psi(x)<t+1\}} \widetilde{g}|\widetilde{f}|_{\omega, h}^{2} e^{-m_{p} \psi} d V_{X, \omega}
$$

for any $g \in \mathcal{C}_{c}\left(Z_{p}^{\circ}\right)$, where $\widetilde{g} \in \mathcal{C}_{c}(X)$ is a continuous extension of $g$ and $\widetilde{f}$ a smooth extension of $f$ on $X$ such that $\tilde{f}-f \in \mathcal{I}\left(m_{p} \psi\right) \otimes_{\mathcal{O}_{X}} \mathcal{C}^{\infty}$ (this measure again has a smooth positive density on a Zariski open set in $Z_{p}^{\circ}$, and does not depend on the choices of $\widetilde{f}$ and $\widetilde{g}$ ). We extend the measure as being 0 on $Y_{\text {red }}^{\left(m_{p}\right)} \backslash Z_{p}$, since $\left.\mathcal{I}\left(e^{-m_{p-1} \psi}\right) / \mathcal{I}\left(e^{-m_{p} \psi}\right)\right)$ has support in $Z_{p}^{\circ} \subset Z_{p}$. In this context, we introduce the following natural definition.

Definition 1.6. We define the restricted multiplied ideal sheaf

$$
\mathcal{I}^{\prime}\left(e^{-m_{p-1} \psi}\right) \subset \mathcal{I}\left(e^{-m_{p-1} \psi}\right)
$$

to be the set of germs $F \in \mathcal{I}\left(e^{-m_{p-1} \psi}\right)_{x} \subset \mathcal{O}_{X, x}$ such that there exists a neighborhood $U$ of $x$ satisfying

$$
\int_{Y^{\left(m_{p}\right)} \cap U}\left|J^{m_{p}} F\right|_{\omega, h}^{2} d V_{Y^{\left(m_{p}\right)}, \omega}[\psi]<+\infty .
$$

This is a coherent ideal sheaf that contains $\mathcal{I}\left(e^{-m_{p} \psi}\right)$. Both of the inclusions

$$
\mathcal{I}\left(e^{-m_{p} \psi}\right) \subset \mathcal{I}^{\prime}\left(e^{-m_{p-1} \psi}\right) \subset \mathcal{I}\left(e^{-m_{p-1} \psi}\right)
$$

can be strict (even for $p=1$ ).

One of the geometric consequences is the following "quantitative" surjectivity statement, which is the analogue of Theorem 1.4 for the case when the first non trivial jumping number $m_{1}=1$ is replaced by a higher jumping number $m_{p}$.

Theorem 1.7. With the above notation and in the general setting of Theorem 1.4 (but without the hypothesis that the quasi-psh function $\psi$ has log canonical singularities), let $0=m_{0}<m_{1}<$ $m_{2}<\ldots<m_{p}<\ldots$ be the jumping numbers of $\psi$. Assume that

$$
\Theta_{E, h}+i\left(m_{p}+\alpha \delta\right) \partial \bar{\partial} \psi \otimes \operatorname{Id}_{E} \geq 0
$$

is Nakano semipositive for $\alpha=0,1$ and some $\delta>0$.

(a) Let

$$
f \in H^{0}\left(Y^{\left(m_{p}\right)}, \mathcal{O}_{X}\left(K_{X} \otimes E\right) \otimes \mathcal{I}^{\prime}\left(e^{-m_{p-1} \psi}\right) / \mathcal{I}\left(e^{-m_{p} \psi}\right)\right)
$$

be a section such that

$$
\int_{Y^{\left(m_{p}\right)}}\left|J^{m_{p}} f\right|_{\omega, h}^{2} d V_{Y^{\left(m_{p}\right)}, \omega}[\psi]<+\infty
$$

Then there exists a global section

$$
F \in H^{0}\left(X, \mathcal{O}_{X}\left(K_{X} \otimes E\right) \otimes \mathcal{I}^{\prime}\left(e^{-m_{p-1} \psi}\right)\right)
$$

which maps to $f$ under the morphism $\mathcal{I}^{\prime}\left(e^{-m_{p-1} \psi}\right) \rightarrow \mathcal{I}\left(e^{-m_{p-1} \psi}\right) / \mathcal{I}\left(e^{-m_{p} \psi}\right)$, such that

$$
\int_{X} \gamma(\delta \psi)|F|_{\omega, h}^{2} e^{-m_{p} \psi} d V_{X, \omega}[\psi] \leq \frac{34}{\delta} \int_{Y^{\left(m_{p}\right)}}\left|J^{m_{p}} f\right|_{\omega, h}^{2} d V_{Y^{\left(m_{p}\right)}, \omega}[\psi] .
$$

(b) The restriction morphism

$$
\begin{aligned}
H^{0}\left(X, \mathcal{O}_{X}(\right. & \left.\left.K_{X} \otimes E\right) \otimes \mathcal{I}^{\prime}\left(e^{-m_{p-1} \psi}\right)\right) \\
& \rightarrow H^{0}\left(Y^{\left(m_{p}\right)}, \mathcal{O}_{X}\left(K_{X} \otimes E\right) \otimes \mathcal{I}^{\prime}\left(e^{-m_{p-1} \psi}\right) / \mathcal{I}\left(e^{-m_{p} \psi}\right)\right)
\end{aligned}
$$

is surjective.

If $E$ is a line bundle and $h$ a singular hermitian metric on $E$, a similar result can be obtained by approximating $h$. However, the $L^{2}$ estimates then require to incorporate $h$ into the definition of the multiplier ideals, as in Theorem 1.1 (see [Dem15a]). Hosono [Hos17a] has shown that one can obtain again an optimal $L^{2}$ estimate in the situation of Theorem 1.7, when $\mathcal{I}\left(e^{-m_{p} \psi}\right)$ is a power of the reduced ideal of $Y$. 
Question 1.8. It would be interesting to know whether Theorem 1.1 can be strengthened by suitable $L^{2}$ estimates, without making undue additional hypotheses on the section $f$ to extend. The main difficulty is already to define the norm of jets when there is more than one jump number involved. Some sort of "Cauchy inequality" for jets would be needed in order to derive the successive jet norms from a known global $L^{2}$ estimate for a holomorphic section defined on the whole of $X$. We do not know how to proceed further at this point.

\section{Bochner-KodAiRA ESTIMATE With APPROXIMATION}

The crucial idea of the proof is to prove the results (say, in the form of the surjectivity statement), only up to approximation. This is done by solving a $\bar{\partial}$-equation

$$
\bar{\partial} u_{\varepsilon}+w_{\varepsilon}=v
$$

where the right hand side $v$ is given and $w_{\varepsilon}$ is an error term such that $\left\|w_{\varepsilon}\right\|=O\left(\varepsilon^{a}\right)$ as $\varepsilon \rightarrow 0$, for some constant $a>0$. A twisted Bochner-Kodaira-Nakano identity introduced by Donnelly and Fefferman [DF83, and Ohsawa and Takegoshi OT87 is used for that purpose. The technology goes back to the fundamental work of Bochner ([Boc48]), Kodaira ([Kod53a, Kod53b, Kod54]), Akizuki-Nakano ([AN54], Nak55]), Kohn ([FK]), Andreotti-Vesentini ([AV65]), Hörmander ([Hör65, Hör66]). The version we need uses in an essential way an additional correction term, so as to allow a weak positivity hypothesis. It can be stated as follows.

Proposition 2.1. (see Dem15b, Prop. 3.12]) Let $X$ be a complete Kähler manifold equipped with a (non necessarily complete) Kähler metric $\omega$, and let $(E, h)$ be a Hermitian vector bundle over $X$. Assume that there are smooth and bounded functions $\eta, \lambda>0$ on $X$ such that the curvature operator

$$
B=B_{E, h, \omega, \eta, \lambda}^{n, q}=\left[\eta \Theta_{E, h}-i \partial \bar{\partial} \eta-i \lambda^{-1} d \partial \eta \wedge \bar{\partial} \eta, \Lambda_{\omega}\right] \in C^{\infty}\left(X, \operatorname{Herm}\left(\Lambda^{n, q} T_{X}^{*} \otimes E\right)\right)
$$

satisfies $B+\varepsilon I>0$ for some $\varepsilon>0$ (so that $B$ can be just semi-positive or even slightly negative; here $I$ is the identity endomorphism). Given a section $v \in L^{2}\left(X, \Lambda^{n, q} T_{X}^{*} \otimes E\right)$ such that $\bar{\partial} v=0$ and

$$
M(\varepsilon):=\int_{X}\left\langle(B+\varepsilon I)^{-1} v, v\right\rangle d V_{X, \omega}<+\infty,
$$

there exists an approximate solution $f_{\varepsilon} \in L^{2}\left(X, \Lambda^{n, q-1} T_{X}^{*} \otimes E\right)$ and a correction term $w_{\varepsilon} \in$ $L^{2}\left(X, \Lambda^{n, q} T_{X}^{*} \otimes E\right)$ such that $\bar{\partial} u_{\varepsilon}=v-w_{\varepsilon}$ and

$$
\int_{X}(\eta+\lambda)^{-1}\left|u_{\varepsilon}\right|^{2} d V_{X, \omega}+\frac{1}{\varepsilon} \int_{X}\left|w_{\varepsilon}\right|^{2} d V_{X, \omega} \leq M(\varepsilon) .
$$

Moreover, if $v$ is smooth, then $u_{\varepsilon}$ and $w_{\varepsilon}$ can be taken smooth.

In our situation, the main part of the solution, namely $u_{\varepsilon}$, may very well explode as $\varepsilon \rightarrow 0$. In order to show that the equation $\bar{\partial} u=v$ can be solved, it is therefore needed to check that the space of coboundaries is closed in the space of cocycles in the Fréchet topology under consideration (here, the $L_{\text {loc }}^{2}$ topology), in other words, that the related cohomology group $H^{q}(X, \mathcal{F})$ is Hausdorff. In this respect, the fact of considering $\bar{\partial}$-cohomology of smooth forms equipped with the $C^{\infty}$ topology on the one hand, or cohomology of forms $u \in L_{\text {loc }}^{2}$ with $\bar{\partial} u \in L_{\text {loc }}^{2}$ on the other hand, yields the same topology on the resulting cohomology group $H^{q}(X, \mathcal{F})$. This comes from the fact that both complexes yield fine resolutions of the same coherent sheaf $\mathcal{F}$, and the topology of $H^{q}(X, \mathcal{F})$ can also be obtained by using Čech cochains with respect to a Stein covering $\mathcal{U}$ of $X$. The required Hausdorff property then comes from the following well known fact.

Lemma 2.2. Let $X$ be a holomorphically convex complex space and $\mathcal{F}$ a coherent analytic sheaf over $X$. Then all cohomology groups $H^{q}(X, \mathcal{F})$ are Hausdorff with respect to their natural topology (induced by the Fréchet topology of local uniform convergence of holomorphic cochains). 
In fact, the Remmert reduction theorem implies that $X$ admits a proper holomorphic map $\pi: X \rightarrow S$ onto a Stein space $S$, and Grauert's direct image theorem shows that all direct images $R^{q} \pi_{*} \mathcal{F}$ are coherent sheaves on $S$. Now, as $S$ is Stein, Leray's theorem combined with Cartan's theorem B tells us that we have an isomorphism $H^{q}(X, \mathcal{F}) \simeq H^{0}\left(S, R^{q} \pi_{*} \mathcal{F}\right)$. More generally, if $U \subset S$ is a Stein open subset, we have

$$
H^{q}\left(\pi^{-1}(U), \mathcal{F}\right) \simeq H^{0}\left(U, R^{q} \pi_{*} \mathcal{F}\right)
$$

and when $U \Subset S$ is relatively compact, it is easily seen that this a topological isomorphism of Fréchet spaces since both sides are $\mathcal{O}_{S}(U)$ modules of finite type and can be seen as a Fréchet quotient of some direct sum $\mathcal{O}_{S}(U)^{\oplus N}$ by looking at local generators and local relations of $R^{q} \pi_{*} \mathcal{F}$. Therefore $H^{q}(X, \mathcal{F}) \simeq H^{0}\left(S, R^{q} \pi_{*} \mathcal{F}\right)$ is a topological isomorphism and the space of sections in the right hand side is a Fréchet space. In particular, $H^{q}(X, \mathcal{F})$ is Hausdorff.

\section{Sketch of Proof of the Extension theorem}

The reader may consult [Dem15b] and [CDM17] for more details. After possibly shrinking $X$ into a relatively compact holomorphically convex open subset $X^{\prime}=\pi^{-1}\left(S^{\prime}\right) \Subset X$, we can suppose that $\delta>0$ is a constant and that $\psi \leq 0$ (otherwise subtract a large constant to $\psi$ ). As $\pi: X \rightarrow S$ is proper, we can also assume that $X$ admits a finite Stein covering $\mathcal{U}=\left(U_{i}\right)$. Any cohomology class in

$$
H^{q}\left(Y, \mathcal{O}_{X}\left(K_{X} \otimes E\right) \otimes \mathcal{I}(h) / \mathcal{I}\left(h e^{-\psi}\right)\right)
$$

is represented by a holomorphic Cech $q$-cocycle with respect to the covering $\mathcal{U}$

$$
\left(c_{i_{0} \ldots i_{q}}\right), \quad c_{i_{0} \ldots i_{q}} \in H^{0}\left(U_{i_{0}} \cap \ldots \cap U_{i_{q}}, \mathcal{O}_{X}\left(K_{X} \otimes E\right) \otimes \mathcal{I}(h) / \mathcal{I}\left(h e^{-\psi}\right)\right) .
$$

By the standard sheaf theoretic isomorphisms with Dolbeault cohomology (cf. e.g. [Dem-e-book]), this class is represented by a smooth $(n, q)$-form

$$
f=\sum_{i_{0}, \ldots, i_{q}} c_{i_{0} \ldots i_{q}} \rho_{i_{0}} \bar{\partial} \rho_{i_{1}} \wedge \ldots \bar{\partial} \rho_{i_{q}}
$$

by means of a partition of unity $\left(\rho_{i}\right)$ subordinate to $\left(U_{i}\right)$. This form is to be interpreted as a form on the (non reduced) analytic subvariety $Y$ associated with the ideal sheaf $\mathcal{J}=\mathcal{I}\left(h e^{-\psi}\right): \mathcal{I}(h)$ and the structure sheaf $\mathcal{O}_{Y}=\mathcal{O}_{X} / \mathcal{J}$. We get an extension as a smooth (no longer $\bar{\partial}$-closed) $(n, q)$-form on $X$ by taking

$$
\widetilde{f}=\sum_{i_{0}, \ldots, i_{q}} \widetilde{c}_{i_{0} \ldots i_{q}} \rho_{i_{0}} \bar{\partial} \rho_{i_{1}} \wedge \ldots \bar{\partial} \rho_{i_{q}}
$$

where $\widetilde{c}_{i_{0} \ldots i_{q}}$ is an extension of $c_{i_{0} \ldots i_{q}}$ from $U_{i_{0}} \cap \ldots \cap U_{i_{q}} \cap Y$ to $U_{i_{0}} \cap \ldots \cap U_{i_{q}}$. Without loss of generality, we can assume that $\psi$ admits a discrete sequence of "jumping numbers"

$$
0=m_{0}<m_{1}<\cdots<m_{p}<\cdots \quad \text { such that } \mathcal{I}(m \psi)=\mathcal{I}\left(m_{p} \psi\right) \text { for } m \in\left[m_{p}, m_{p+1}[.\right.
$$

Since $\psi$ is assumed to have analytic singularities, this follows from using a log resolution of singularities, thanks to the Hironaka desingularization theorem (by the much deeper result of GZ15b on the strong openness conjecture, one could even possibly eliminate the assumption that $\psi$ has analytic singularities). We fix here $p$ such that $m_{p} \leq 1<m_{p+1}$, and in the notation of Dem15b, we let $Y=Y^{\left(m_{p}\right)}$ be defined by the non necessarily reduced structure sheaf $\mathcal{O}_{Y}=\mathcal{O}_{X} / \mathcal{I}\left(e^{-\psi}\right)=\mathcal{O}_{X} / \mathcal{I}\left(e^{-m_{p} \psi}\right)$.

We now explain the choice of metrics and auxiliary functions $\eta, \lambda$ for the application of Proposition 2.1, following the arguments of [Dem15b, proof of th. 2.14, p. 217]. Let $t \in \mathbb{R}^{-}$ and let $\chi_{t}$ be the negative convex increasing function defined in [Dem15b, $(5.8 *)$, p. 211]. Put $\eta_{t}:=1-\delta \cdot \chi_{t}(\psi)$ and $\lambda_{t}:=2 \delta \frac{\left(\chi_{t}^{2}(\psi)\right)^{2}}{\chi_{t}^{\prime \prime}(\psi)}$. We set

$$
\begin{aligned}
R_{t} & :=\eta_{t}\left(\Theta_{E, h}+i \partial \bar{\partial} \psi\right)-i \partial \bar{\partial} \eta_{t}-\lambda_{t}^{-1} i \partial \eta_{t} \wedge \bar{\partial} \eta_{t} \\
& =\eta_{t}\left(\Theta_{E, h}+\left(1+\delta \eta_{t}^{-1} \chi_{t}^{\prime}(\psi)\right) i \partial \bar{\partial} \psi\right)+\frac{\delta \cdot \chi_{t}^{\prime \prime}(\psi)}{2} i \partial \psi \wedge \bar{\partial} \psi
\end{aligned}
$$


Note that $\chi_{t}^{\prime \prime}(\psi) \geq \frac{1}{8}$ on $W_{t}=\{t<\psi<t+1\}$. The curvature assumption (1.4) implies

$$
\Theta_{E, h}+\left(1+\delta \eta_{t}^{-1} \chi_{t}^{\prime}(\psi)\right) i \partial \bar{\partial} \psi \geq 0 \quad \text { on } X .
$$

As in Dem15b], we find

$$
R_{t} \geq 0 \quad \text { on } X
$$

and

$$
R_{t} \geq \frac{\delta}{16} i \partial \psi \wedge \bar{\partial} \psi \quad \text { on } \quad W_{t}=\{t<\psi<t+1\}
$$

Let $\theta:[-\infty,+\infty[\rightarrow[0,1]$ be a smooth non increasing real function satisfying $\theta(x)=1$ for $x \leq 0, \theta(x)=0$ for $x \geq 1$ and $\left|\theta^{\prime}\right| \leq 2$. By using a blowing up process, one can reduce the situation to the case where $\psi$ has divisorial singularities. Then we still have

$$
\Theta_{E, h}+\left(1+\delta \eta_{t}^{-1} \chi_{t}^{\prime}(\psi)\right)(i \partial \bar{\partial} \psi)_{\mathrm{ac}} \geq 0 \quad \text { on } X,
$$

where $(i \partial \bar{\partial} \psi)_{\mathrm{ac}}$ is the absolutely continuous part of $i \partial \bar{\partial} \psi$. The regularization techniques of [DPS01] and [Dem15a, Th. 1.7, Remark 1.11] produce a family of singular metrics $\left\{h_{t, \varepsilon}\right\}_{k=1}^{+\infty}$ which are smooth in the complement $X \backslash Z_{t, \varepsilon}$ of an analytic set, such that $\mathcal{I}\left(h_{t, \varepsilon}\right)=\mathcal{I}(h)$, $\mathcal{I}\left(h_{t, \varepsilon} e^{-\psi}\right)=\mathcal{I}\left(h e^{-\psi}\right)$ and

$$
\Theta_{E, h_{t, \varepsilon}}+\left(1+\delta \eta_{t}^{-1} \chi_{t}^{\prime}(\psi)\right) i \partial \bar{\partial} \psi \geq-\frac{1}{2} \varepsilon \omega \quad \text { on } X .
$$

The additional error term $-\frac{1}{2} \varepsilon \omega$ is irrelevant when we use Proposition 2.1, as it is absorbed by taking the hermitian operator $B+\varepsilon I$. Therefore for every $t \in \mathbb{R}^{-}$, with the adjustment $\varepsilon=e^{\alpha t}$, $\alpha \in] 0, m_{p+1}-1\left[\right.$, we can find a singular metric $h_{t}=h_{t, \varepsilon}$ which is smooth in the complement $X \backslash Z_{t}$ of an analytic set, such that $\mathcal{I}\left(h_{t}\right)=\mathcal{I}(h), \mathcal{I}\left(h_{t} e^{-\psi}\right)=\mathcal{I}\left(h e^{-\psi}\right)$ and $h_{t} \uparrow h$ as $t \rightarrow-\infty$. We now apply the $L^{2}$ estimate of Proposition 2.1 and observe that $X \backslash Z_{t}$ is complete Kähler (at least after we shrink $X$ a little bit as $X^{\prime}=\pi^{-1}\left(S^{\prime}\right)$, cf. [Dem82]). As a consequence, one can find sections $u_{t}, w_{t}$ satisfying

$$
\bar{\partial} u_{t}+w_{t}=v_{t}:=\bar{\partial}(\theta(\psi-t) \cdot \tilde{f})
$$

and

$$
\begin{gathered}
\int_{X}\left(\eta_{t}+\lambda_{t}\right)^{-1}\left|u_{t}\right|_{\omega, h_{t}}^{2} e^{-\psi} d V_{X, \omega}+\frac{1}{\varepsilon} \int_{X}\left|w_{t}\right|_{\omega, h_{t}}^{2} e^{-\psi} d V_{X, \omega} \\
\leq \int_{X}\left\langle\left(R_{t}+\varepsilon I\right)^{-1} v_{t}, v_{t}\right\rangle_{\omega, h_{t}} e^{-\psi} d V_{X, \omega} .
\end{gathered}
$$

One of the main consequence of (3.3) and (3.5) is that, for $\varepsilon=e^{\alpha t}$ and $\alpha$ well chosen, one can infer that the error term satisfies

$$
\lim _{t \rightarrow-\infty} \int_{X}\left|w_{t}\right|_{\omega, h_{t}}^{2} e^{-\psi} d V_{X, \omega}=0 .
$$

One difficulty, however, is that $L^{2}$ sections cannot be restricted in a continuous way to a subvariety. In order to overcome this problem, we play again the game of returning to Čech cohomology by solving inductively $\bar{\partial}$-equations for $w_{t}$ on $U_{i_{0}} \cap \ldots \cap U_{i_{k}}$, until we reach an equality

$$
\bar{\partial}\left(\theta(\psi-t) \cdot \tilde{f}-\widetilde{u}_{t}\right)=\widetilde{w}_{t}:=-\sum_{i_{0}, \ldots, i_{q-1}} s_{t, i_{0} \ldots i_{q}} \bar{\partial} \rho_{i_{0}} \wedge \bar{\partial} \rho_{i_{1}} \wedge \ldots \bar{\partial} \rho_{i_{q}}
$$

with holomorphic sections $s_{t, I}=s_{t, i_{0} \ldots i_{q}}$ on $U_{I}=U_{i_{0}} \cap \ldots \cap U_{i_{q}}$, such that

$$
\lim _{t \rightarrow-\infty} \int_{U_{I}}\left|s_{t, I}\right|_{\omega, h_{t}}^{2} e^{-\psi} d V_{X, \omega}=0 .
$$

Then the right hand side of (3.6) is smooth, and more precisely has coefficients in the sheaf $\mathcal{C}^{\infty} \otimes_{\mathcal{O}} \mathcal{I}\left(h e^{-\psi}\right)$, and $\widetilde{w}_{t} \rightarrow 0$ in $C^{\infty}$ topology. A priori, $\widetilde{u}_{t}$ is an $L^{2}(n, q)$-form equal to $u_{t}$ 
plus a combination $\sum \rho_{i} s_{t, i}$ of the local solutions of $\bar{\partial} s_{t, i}=w_{t}$, plus $\sum \rho_{i} s_{t, i, j} \wedge \bar{\partial} \rho_{j}$ where $\bar{\partial} s_{t, i, j}=s_{t, j}-s_{t, i}$, plus etc $\ldots$, and is such that

$$
\int_{X}\left|\widetilde{u}_{t}\right|_{\omega, h_{t}}^{2} e^{-\psi} d V_{X, \omega}<+\infty
$$

Since $H^{q}\left(X, \mathcal{O}_{X}\left(K_{X} \otimes E\right) \otimes \mathcal{I}\left(h e^{-\psi}\right)\right)$ can be computed with the $L_{\text {loc }}^{2}$ resolution of the coherent sheaf, or alternatively with the $\bar{\partial}$-complex of $(n, \bullet)$-forms with coefficients in $\mathcal{C}^{\infty} \otimes_{\mathcal{O}} \mathcal{I}\left(h e^{-\psi}\right)$, we may assume that $\widetilde{u}_{t} \in \mathcal{C}^{\infty} \otimes_{\mathcal{O}} \mathcal{I}\left(h e^{-\psi}\right)$, after playing again with Čech cohomology. Lemma 2.2 yields a sequence of smooth $(n, q)$-forms $\sigma_{t}$ with coefficients in $\mathcal{C}^{\infty} \otimes_{\mathcal{O}} \mathcal{I}(h)$, such that $\bar{\partial} \sigma_{t}=\widetilde{w}_{t}$ and $\sigma_{t} \rightarrow 0$ in $C^{\infty}$-topology. Then $\widetilde{f}_{t}=\theta(\psi-t) \cdot \widetilde{f}-\widetilde{u}_{t}-\sigma_{t}$ is a $\bar{\partial}$-closed $(n, q)$-form on $X$ with values in $\mathcal{C}^{\infty} \otimes_{\mathcal{O}} \mathcal{I}(h) \otimes \mathcal{O}_{X}(E)$, whose image in $H^{q}\left(X, \mathcal{O}_{X}\left(K_{X} \otimes E\right) \otimes \mathcal{I}(h) / \mathcal{I}\left(h e^{-\psi}\right)\right)$ converges to $\{f\}$ in $C^{\infty}$ Fréchet topology. We conclude by a density argument on the Stein space $S$, by looking at the coherent sheaf morphism

$$
R^{q} \pi_{*}\left(\mathcal{O}_{X}\left(K_{X} \otimes E\right) \otimes \mathcal{I}(h)\right) \rightarrow R^{q} \pi_{*}\left(\mathcal{O}_{X}\left(K_{X} \otimes E\right) \otimes \mathcal{I}(h) / \mathcal{I}\left(h e^{-\psi}\right)\right) .
$$

Proof of the quantitative estimates. We refer again to Dem15b for details. One of the main features of the above qualitative proof is that we have not tried to control the solution $u_{t}$ of our $\bar{\partial}$-equation, in fact we only needed to prove that the error term $w_{t}$ converges to zero. However, to get quantitative $L^{2}$ estimates, we have to pay attention to the $L^{2}$ norm of $u_{t}$. It is under control as $t \rightarrow-\infty$ only when $f$ satisfies the more restrictive condition of being $L^{2}$ with respect to the residue measure $d V_{Y^{\circ}, \omega}[\psi]$. This is the reason why we lose track of the solution when the volume of the measure explodes on $Y_{\text {sing }}$, or when there are several jumps involved in the multiplier ideal sheaves.

\section{Applications of the Ohsawa-Takegoshi extension theorem}

The Ohsawa-Takegoshi extension theorem is a very powerful tool that has many important applications to complex analysis and geometry. We will content ourselves by mentioning only a few statements and references.

4.1. Approximation of plurisubharmonic functions and of closed $(1,1)$-currents. By considering the extension from points (i.e. a 0-dimensional connected subvariety $Y \subset X$ ), even just locally on coordinates balls, one gets a precise Bergman kernel estimate for Hilbert spaces attached to multiples of any plurisubharmonic function. This leads to regularization theorems ([Dem92]) that have many applications, such as the Hard Lefschetz theorem with multiplier ideal sheaves ([DPS01]), or extended vanishing theorems for pseudoeffective line bundles ([Cao14]). The result may consult Dem15a] for a survey of these questions. Another consequence is a very simple and direct proof of Siu's result [Siu74] on the analyticity of sublevel sets of Lelong numbers of closed positive currents.

4.2. Invariance of plurigenera. Around 2000, Siu [Siu02] proved that for every smooth projective deformation $\pi: \mathcal{X} \rightarrow S$ over an irreducible base $S$, the plurigenera $p_{m}(t)=h^{0}\left(X_{t}, K_{X_{t}}^{\otimes m}\right)$ of the fibers $X_{t}=\pi^{-1}(t)$ are constant. The proof relies in an essential way on the OhsawaTakegoshi extension theorem, and was later simplified and generalized by Păun [Pau07]. It is remarkable that no algebraic proof of this purely algebraic result is known!

4.3. Semicontinuity of log singularity exponents. In [DK01, we proved that the log singularity exponent (or $\log$ canonical threshold) $c_{x}(\varphi)$, defined as the supremum of constants $c>0$ such that $e^{-c \varphi}$ is integrable in a neighborhood of a point $x$, is a lower semicontinuous function with respect to the topology of weak convergence on plurisubharmonic functions. Guan and Zhou [GZ15b] recently proved our "strong openness conjecture", namely that the integrability of $e^{-\varphi}$ implies the integrability of $e^{-(1+\varepsilon) \varphi}$ for $\varepsilon>0$ small; later alternative proofs have been exposed in [Hie14] and [Lem14]. 
4.4. Proof of the Suita conjecture. In Blo13 Błocki determined the value of the optimal constant in the Ohsawa-Takegoshi extension theorem, a result that was subsequently generalized by Guan and Zhou GZ15a]. In complex dimension 1, this result implies in its turn a conjecture of N. Suita, stating that for any bounded domain $D$ in $\mathbb{C}$, one has $c_{D}^{2} \leq \pi K_{D}$, where $c_{D}(z)$ is the logarithmic capacity of $\mathbb{C} \backslash D$ with respect to $z \in D$ and $K_{D}$ is the Bergman kernel on the diagonal. Guan and Zhou GZ15a] proved that the equality occurs if ond only if $D$ is conformally equivalent to the disc minus a closed set of inner capacity zero.

\section{REFERENCES}

[AN54] Akizuki, Y., Nakano, S., Note on Kodaira-Spencer's proof of Lefschetz theorems, Proc. Jap. Acad. 30 (1954) 266-272.

[AV65] Andreotti, A., Vesentini, E., Carleman estimates for the Laplace-Beltrami equation in complex manifolds, Publ. Math. I.H.E.S. 25 (1965) 81-130.

[Ber96] Berndtsson, B., The extension theorem of Ohsawa-Takegoshi and the theorem of Donnelly-Fefferman, Ann. Inst. Fourier 14 (1996) 1087-1099.

[BL16] Berndtsson, B., Lempert, L., A proof of the Ohsawa-Takegoshi theorem with sharp estimates, J. Math. Soc. Japan 68 (2016), no. 4, 1461-1472.

[Blo13] Błocki, Z., Suita conjecture and the Ohsawa-Takegoshi extension theorem, Invent. Math. 193 (2013), no. 1, 149-158.

[Boc48] Bochner, S., Curvature and Betti numbers (I) and (II), Ann. of Math. 49 (1948), 379-390; 50 (1949), $77-93$.

[Cao14] Cao, Junyan, Numerical dimension and a Kawamata-Viehweg-Nadel-type vanishing theorem on compact Kähler manifolds, Compositio Mathematica 150 (2014) 1869-1902.

[CDM17] Cao, Junyan, Demailly, J.-P., Matsumura, Shin-ichi, A general extension theorem for cohomology classes on non reduced analytic subspaces, Science China Mathematics, Volume 60, Issue 6, June 2017, 949-962.

[Che11] Chen, Bo-Yong, A simple proof of the Ohsawa-Takegoshi extension theorem, arXiv: math.CV/ 1105.2430.

[Dem82] Demailly, J.-P., Estimations $L^{2}$ pour l'opérateur $\bar{\partial}$ d'un fibré vectoriel holomorphe semi-positif au-dessus d'une variété kählérienne complète, Ann. Sci. École Norm. Sup(4). 15 (1982), 457-511.

[Dem92] Demailly, J.-P., Regularization of closed positive currents and Intersection Theory, J. Alg. Geom. 1 (1992), 361-409.

[Dem97] Demailly, J.-P., On the Ohsawa-Takegoshi-Manivel $L^{2}$ extension theorem, Proceedings of the Conference in honour of the 85th birthday of Pierre Lelong, Paris, September 1997, éd. P. Dolbeault, Progress in Mathematics, Birkhäuser, Vol. 188 (2000), 47-82.

[Dem15a] Demailly, J.-P., On the cohomology of pseudoeffective line bundles, Complex Geometry and Dynamics, The Abel Symposium 2013 held at Trondheim, ed. John Erik Fornæss, Marius Irgens, Erlend Fornæss Wold, Springer-Verlag 2015, 51-99.

[Dem15b] Demailly, J.-P., Extension of holomorphic functions defined on non reduced analytic subvarieties, Advanced Lectures in Mathematics ALM35, the legacy of Bernhard Riemann after one hundred and fifty years, Higher Education Press, Beijing-Boston, 2015, 191-222.

[Dem-e-book] Demailly, J.-P., Complex analytic and differential geometry, e-book available on the web page of the author, https://www-fourier.ujf-grenoble.fr/ demailly/manuscripts/agbook.pdf

[Dem-book] Demailly, J.-P., Analytic methods in algebraic geometry, Surveys of Modern Mathematics, 1. International Press, Somerville, Higher Education Press, Beijing, (2012).

[DHP13] Demailly, J.-P., Hacon, Ch., Păun, M.. Extension theorems, Non-vanishing and the existence of good minimal models. Acta Math. 210 (2013), 203-259.

[DK01] Demailly, J.-P., Kollár, J., Semicontinuity of complex singularity exponents and Kähler-Einstein metrics on Fano orbifolds, Ann. Ec. Norm. Sup. 34 (2001) 525-556.

[DPS01] Demailly, J.-P., Peternell, T., Schneider, M., Pseudo-effective line bundles on compact Kähler manifolds, International Journal of Math. 6 (2001), 689-741.

[DF83] Donnelly, H., Fefferman, C., $L^{2}$-cohomology and index theorem for the Bergman metric, Ann. Math. 118 (1983) 593-618.

[FK] Folland, G.B., Kohn, J.J., The Neumann problem for the Cauchy-Riemann complex, Annals of Mathematics Studies, No. 75. Princeton University Press, Princeton, N.J.; University of Tokyo Press, Tokyo, (1972).

[Fuj13] Fujino, O., A transcendental approach to Kollár's injectivity theorem II, J. Reine Angew. Math. 681 (2013), 149-174.

[FM16] Fujino, O., Matsumura, S.-i., Injectivity theorem for pseudo-effective line bundles and its applications, arXiv:1605.02284v 1 .

[GZ15a] Guan, Qi'an, Zhou, Xiangyu, A solution of an $L^{2}$ extension problem with an optimal estimate and applications, Ann. of Math. (2) 181 (2015), no. 3, 1139-1208.

[GZ15b] Guan Qi'an, Zhou, Xiangyu, A proof of Demailly's strong openness conjecture, Annals of Math. 182 (2015), no. 2, 605-616. 
[Hie14] Pham H. Hiêp, The weighted log canonical threshold, C. R. Math. Acad. Sci. Paris 352 (2014), no. 4, 283-288.

[Hör65] Hörmander, L., $L^{2}$ estimates and existence theorems for the $\bar{\partial}$ operator, Acta Math. 113 (1965), 89-152.

[Hör66] Hörmander, L., An introduction to Complex Analysis in several variables, 1st edition, Elsevier Science Pub., New York, 1966, 3rd revised edition, North-Holland Math. library, Vol 7, Amsterdam (1990).

[Hos17a] Hosono, Genki, The optimal jet $L^{2}$ extension of Ohsawa-Takegoshi type, arXiv: math.CV/1706.08725

[Hos17b] Hosono, Genki, On sharper estimates of Ohsawa-Takegoshi $L^{2}$-extension theorem, arXiv: math.CV/1708.08269.

[Kod53a] Kodaira, K., On cohomology groups of compact analytic varieties with coefficients in some analytic faisceaux, Proc. Nat. Acad. Sci. U.S.A. 39 (1953), 868-872.

[Kod53b] Kodaira, K., On a differential geometric method in the theory of analytic stacks, Proc. Nat. Acad. Sci. U.S.A. 39 (1953), 1268-1273.

[Kod54] Kodaira, K., On Kähler varieties of restricted type, Ann. of Math. 60 (1954), 28-48.

[Lem14] Lempert, L., Modules of square integrable holomorphic germs, in: Analysis Meets Geometry: A Tribute to Mikael Passare, Trends in Mathematics, Springer International Publishing, 2017, 311-333.

[Man93] Manivel, L., Un théorème de prolongement $L^{2}$ de sections holomorphes d'un fibré vectoriel, Math. Zeitschrift 212 (1993), 107-122.

[Mat16] Matsumura, S.-i., An injectivity theorem with multiplier ideal sheaves for higher direct images under Kähler morphisms, arXiv: math.CV/1607.05554v1.

[Mat17] Matsumura, S.-i., An injectivity theorem with multiplier ideal sheaves of singular metrics with transcendental singularities, arXiv: math.CV/1308.2033v4, J. Algebraic Geom., DOI: https://doi.org/10.1090/jag/687, e-pub. August 17, 2017.

[MV07] McNeal, J., Varolin, D., Analytic inversion of adjunction: $L^{2}$ extension theorems with gain, Ann. Inst. Fourier (Grenoble) $5 \mathbf{7}$ (2007), no. 3, 703-718.

[Nak55] Nakano, S., On complex analytic vector bundles, J. Math. Soc. Japan 7 (1955), 1-12.

[Nad89] Nadel, A.M., Multiplier ideal sheaves and Kähler-Einstein metrics of positive scalar curvature, Proc. Nat. Acad. Sci. U.S.A. 86 (1989), 7299-7300 and Annals of Math., 132 (1990), 549-596.

[Nak73] Nakano, S., Vanishing theorems for weakly 1-complete manifolds, Number Theory, Algebraic Geometry and Commutative Algebra, in honor of Y. Akizuki, Kinokuniya, Tokyo (1973), 169-179.

[Nak74] Nakano, S., Vanishing theorems for weakly 1-complete manifolds II, Publ. R.I.M.S., Kyoto Univ. 10 (1974), 101-110.

[Ohs88] Ohsawa, T., On the extension of $L^{2}$ holomorphic functions, II, Publ. RIMS, Kyoto Univ. 24 (1988), $265-275$.

[Ohs94] Ohsawa, T., On the extension of $L^{2}$ holomorphic functions, IV: A new density concept, Mabuchi, T. (ed.) et al., Geometry and analysis on complex manifolds. Festschrift for Professor S. Kobayashi's 60th birthday. Singapore: World Scientific, (1994), 157-170.

[Ohs95] Ohsawa, T., On the extension of $L^{2}$ holomorphic functions, III : negligible weights, Math. Zeitschrift 219 (1995), 215-225.

[Ohs01] Ohsawa, T., On the extension of $L^{2}$ holomorphic functions, $V$ : Effects of generalization, Nagoya Math. J. 161 (2001), 1-21, erratum: Nagoya Math. J. 163 (2001), 229.

[Ohs03] Ohsawa, T., On the extension of $L^{2}$ holomorphic functions, VI: A limiting case, Explorations in complex and Riemannian geometry, Contemp. Math., 332, Amer. Math. Soc., Providence, RI, 2003, 235-239.

[Ohs04] T. Ohsawa, On a curvature condition that implies a cohomology injectivity theorem of Kollár-Skoda type, Publ. Res. Inst. Math. Sci. 41 (2005), no. 3, 565-577.

[Ohs05] Ohsawa, T., $L^{2}$ extension theorems - backgrounds and a new result, Finite or infinite dimensional complex analysis and applications, Kyushu University Press, Fukuoka, 2005, 261-274.

[OT87] Ohsawa, T., Takegoshi, K., On the extension of $L^{2}$ holomorphic functions, Math. Zeitschrift 195 (1987), $197-204$

[Pau07] Păun, M., Siu's invariance of plurigenera: a one-tower proof, J. Differential Geom. 76 (2007) $485-493$.

[Pop05] Popovici, D., $L^{2}$ extension for jets of holomorphic sections of a Hermitian line bundle, Nagoya Math. J. 180 (2005), 1-34.

[Siu74] Siu, Y.-T., Analyticity of sets associated to Lelong numbers and the extension of closed positive currents, Invent. Math. 27 (1974), 53-156.

[Siu02] Siu, Y.-T., Extension of twisted pluricanonical sections with plurisubharmonic weight and invariance of semipositively twisted plurigenera for manifolds not necessarily of general type, Complex Geometry (Göttingen, 2000), Springer, Berlin, 2002, 223-277.

[Var10] Varolin, D., Three variations on a theme in complex analytic geometry, Analytic and Algebraic Geometry, IAS/Park City Math. Ser. 17, Amer. Math. Soc., (2010), 183-294.

(*) Institut Fourier, Université Grenoble Alpes, 100 rue des Maths, 38610 Gières, France e-mail: jean-pierre.demailly@univ-grenoble-alpes.fr 